Nonlinear Processes in Geophysics (2002) 9: 221-235

Nonlinear Processes

in Geophysics

(C)European Geophysical Society 2002

\title{
Higher-order Korteweg-de Vries models for internal solitary waves in a stratified shear flow with a free surface
}

\author{
R. Grimshaw ${ }^{1}$, E. Pelinovsky ${ }^{2}$, and O. Poloukhina ${ }^{2}$ \\ ${ }^{1}$ Department of Mathematical Sciences, Loughborough University, Loughborough, UK \\ ${ }^{2}$ Laboratory of Hydrophysics and Nonlinear Acoustics, Institute of Applied Physics and Department of Applied Mathematics, \\ Nizhny Novgorod Technical University, Nizhny Novgorod, Russia
}

Received: 3 May 2001 - Accepted: 24 August 2001

\begin{abstract}
A higher-order extension of the familiar Korteweg-de Vries equation is derived for internal solitary waves in a density- and current-stratified shear flow with a free surface. All coefficients of this extended Kortewegde Vries equation are expressed in terms of integrals of the modal function for the linear long-wave theory. An illustrative example of a two-layer shear flow is considered, for which we discuss the parameter dependence of the coefficients in the extended Korteweg-de Vries equation.
\end{abstract}

\section{Introduction}

The Korteweg-de Vries (KdV) equation is a well-known model for the description of nonlinear long internal waves in a fluid stratified by both density and current. The steady-state version of this equation was produced by Long (1956), while Benney (1966) gave the integral expressions for the determination of the coefficients of the Korteweg-de Vries equation for waves in a fluid with arbitrary density- and currentstratification. The next step was due to Lee and Beardsley (1974) who indicated the asymptotic procedure needed to produce higher-order Korteweg-de Vries equations based on two small parameters representing dispersion and nonlinearity. More detailed information has been obtained for interfacial waves in a two-layer fluid, and in particular, Kakutani and Yamasaki (1978) found the coefficient of the cubic nonlinear term in an implicit form, and showed its importance in certain special conditions (i.e. the pycnocline lies close to the mid-depth of the fluid), in which case the quadratic and cubic nonlinear terms are of the same order. Due to the negative sign of the coefficient of the cubic nonlinear term, this situation implies that there is an upper limit for the solitary wave amplitude. Then all nonlinear-dispersive coefficients for all second-order terms were found for a two-layer fluid (Koop and Butler, 1981), and solutions of the resulting extended

Correspondence to: R. Grimshaw

(r.h.j.grimshaw@lboro.ac.uk)
Korteweg-de Vries equation were compared with laboratory experiments of internal solitary waves. A more detailed analysis of the properties of the steady-state solitary waves in a fluid with arbitrary density and current stratification, valid to the second order of an asymptotic expansion was reported by Gear and Grimshaw (1983). They calculated the shape of the internal solitary wave and its speed for different models of the fluid stratification.

A lot of data of internal solitary waves has been obtained in the last twenty years by remote sensing and in situ measurements. The necessity to explain this data has induced an interest in developing models for unsteady internal solitary waves in an ocean with realistic stratification in both density and current, which may also vary horizontally. The first step was the introduction of a variable-coefficient Kortewegde Vries equation (Pelinovsky et al., 1994; Grimshaw, 1997). Some calculations of the coefficients of this equation showed that the coefficient of the quadratic nonlinear term will typically change its sign in the coastal zone (see, for instance, Holloway et al., 1997), and, therefore, the contribution of higher-order terms (in particluar, the cubic nonlinear term) should be important. General integral expressions for the coefficients of these higher-order terms for the case of a continuous density-stratification (in the Boussinesq approximation) were produced by Lamb and Yan (1996), and then by Pelinovsky, Poloukhina, Lamb (2000) with the addition of a shear flow, (but also still in the Boussinesq approximation). These expressions are quite complicated, and their signs are not evident without extensive calculation. Consequently, the simpler expressions for a two-layer fluid (Kakutani and Yamasaki, 1978; Koop and Butler, 1981) have usually been used to estimate the signs and the value of the coefficients in the evolution equation. Grimshaw et al. (1997) and Talipova et al. (1999) calculated the coefficient of the cubic nonlinear term for a three-layer fluid with a constant buoyancy frequency in an each layer (but in the Boussinesq approximation) and showed that it may have either sign depending on the layer locations. Such an variation in the sign of the coefficient of the cubic nonlinear term was also obtained for 
the real stratification of a shelf zone (Holloway et al., 1999).

The goal of this paper is to obtain a higher-order Korteweg-de Vries equation for internal waves in an arbitrary density- and current-stratified fluid, without using the Boussinesq approximation, and also taking into account the free surface. An explicit example of a two-layer shear flow will be considered to obtain the coefficients explicitly, so that we are then able to analyse them for different parameter settings.

\section{Governing equations}

The governing equations are those for a two-dimensional flow in an inviscid, incompressible and density-stratified fluid. In Eulerian coordinates they are,

$\rho \frac{d u}{d t}+\frac{\partial p}{\partial x}=0$

$\rho \frac{d w}{d t}+\frac{\partial p}{\partial z}+\rho g=0$,

$\frac{d \rho}{d t}=0$,

$u_{x}+w_{z}=0$,

where $\{u, w\}$ is the fluid velocity, $\rho$ is the fluid density, $p$ is the pressure, $g$ is gravitational acceleration, $\{x, z\}$ are the spatial coordinates (horizontal and vertical) and $d / d t$ is the convective time derivative.

The fluid is bounded below by the rigid boundary $z=-h$ and above by the free surface whose equilibrium position is at $z=0$. Thus, boundary conditions for Eqs. (1)-(4) have the form,

$w=\left.0\right|_{z=-h}$,

$p=\left.0\right|_{z=\xi(x, t)}$,

$w=\frac{\partial \xi}{\partial t}+\left.u \frac{\partial \xi}{\partial x}\right|_{z=\xi}$,

where $\xi(x, t)$ is the vertical displacement of the free surface.

Let us express these equations in non-dimensional form based on the dimensionless variables,

$(x, z, t)=\left(h_{0} \tilde{x}, h_{0} \tilde{z}, \frac{1}{N_{0}} \widetilde{t}\right)$,

$(u, w)=h_{0} N_{0}(\widetilde{u}, \widetilde{w})$,

$(\rho, p)=\left(\bar{\rho} \widetilde{\rho}, \bar{\rho} g h_{0} \tilde{p}\right)$,

where $h_{0}$ is a typical length scale, $N_{0}$ is a typical value of the buoyancy frequency, and $\bar{\rho}$ is typical density value; note that for a layered fluid, $N_{0}$ is interpreted as a measure of a typical density jump, i.e. $N_{0}^{2}=g \Delta \bar{\rho} / h_{0} \bar{\rho}$.

After dropping the tilde superscripts Eqs. (3) and (4) are unchanged, as are the boundary conditions, while Eqs. (1) and (2) take the form,

$\rho \frac{d u}{d t}+\frac{1}{\sigma} \frac{\partial p}{\partial x}=0$

$\rho \frac{d w}{d t}+\frac{1}{\sigma}\left(\frac{\partial p}{\partial z}+\rho\right)=0$, where $\sigma=N_{0}^{2} h_{0} / g$. This parameter is small for oceanic conditions, and $\sigma \rightarrow 0$ defines the Boussinesq approximation.

Thus, we shall use Eqs. (1)-(4), the kinematic condition (7), and the boundary conditions (5) and (6) to study internal solitary waves.

\section{Semi-Lagrangian form of the governing equations}

First we introduce $\zeta(x, z, t)$ as the vertical displacement of a fluid particle from its rest position, so that

$w=d \zeta / d t$

We suppose that the density of the fluid in the rest state is given by $\rho_{0}(z)$. Then in the disturbed state, $\rho(x, z, t)=$ $\rho_{0}(z-\zeta(x, z, t))$, so that Eq. (3) is now satisfied. Also, it is convenient to express the pressure in the form,

$p(x, z, t)=-\int_{0}^{z} \rho_{0}\left(z^{\prime}\right) d z^{\prime}+\sigma q(x, z, t)$.

Then we introduce the isopycnal (Lagrangian) coordinate,

$y=z-\zeta(x, z, t)$.

Thus, the density $\rho(x, z, t)=\rho_{0}(y)$ and is fixed in this representation. To determine how the equations transform, when we change the $(x, z, t)$ coordinates to $(x, y, t)$, let

$f(x, z, t)=F(x, y, t)$.

Therefore, we have the relations,

$\frac{\partial f}{\partial x}=\frac{\partial F}{\partial x}-\frac{\partial F}{\partial y} \frac{\partial \zeta}{\partial x}$,

$\frac{\partial f}{\partial t}=\frac{\partial F}{\partial t}-\frac{\partial F}{\partial y} \frac{\partial \zeta}{\partial t}$

$\frac{\partial f}{\partial z}=\frac{\partial F}{\partial y}\left(1-\frac{\partial \zeta}{\partial z}\right)$.

Consequently,

$\frac{d f}{d t}=\frac{\partial F}{\partial t}+u \frac{\partial F}{\partial x}$.

In particular, if we let

$\zeta(x, z, t)=\eta(x, y, t)$

then

$\zeta_{z}=\frac{\eta_{y}}{1+\eta_{y}}, \quad \zeta_{x}=\frac{\eta_{x}}{1+\eta_{y}}, \quad \zeta_{t}=\frac{\eta_{t}}{1+\eta_{y}}$,

where the indices denote partial derivatives. Then, using Eqs. (16)-(19), we can rewrite Eqs. (11), (12), and (4), so that, on omitting the formal distinction between $f$ and $F$ we obtain,

$\rho_{0}(y)\left(\frac{\partial u}{\partial t}+u \frac{\partial u}{\partial x}\right)+\frac{\partial q}{\partial x}-\frac{1}{1+\frac{\partial \eta}{\partial y}} \frac{\partial q}{\partial y} \frac{\partial \eta}{\partial x}=0$ 


$$
\begin{gathered}
\rho_{0}(y)\left(\frac{\partial w}{\partial t}+u \frac{\partial w}{\partial x}\right)+\frac{1}{1+\frac{\partial \eta}{\partial y}} \frac{\partial q}{\partial y} \\
+\frac{1}{\sigma}\left[\rho_{0}(y)-\rho_{0}(y+\eta)\right]=0 \\
\frac{\partial u}{\partial x}+\frac{\partial w}{\partial y}-\frac{1}{1+\frac{\partial \eta}{\partial y}}\left(\frac{\partial u}{\partial y} \frac{\partial \eta}{\partial x}+\frac{\partial w}{\partial y} \frac{\partial \eta}{\partial y}\right)=0 .
\end{gathered}
$$

Also, the kinematic condition (7) becomes

$w=\frac{\partial \eta}{\partial t}+u \frac{\partial \eta}{\partial x}$

In these new coordinates the boundary conditions (5) and (6) become, respectively,

$\eta=\left.0\right|_{y=-h}$,

$\int_{0}^{\eta} \rho_{0}\left(y^{\prime}\right) d y^{\prime}=\left.\sigma q\right|_{y=0}$

The set of Eqs. (20), (21) and (22) with the kinematic condition (23) can be reduced to two equations for $u(x, y, t)$, $\eta(x, y, t)$. The first is

$$
\begin{gathered}
\frac{\partial}{\partial y}\left\{\rho_{0}(y)\left(\frac{\partial u}{\partial t}+u \frac{\partial u}{\partial x}\right)\right\}-\rho_{0}(y) N^{2}(y) \frac{\partial \eta}{\partial x} \\
-\frac{\partial}{\partial x}\left\{\rho_{0}(y)\left(\frac{\partial}{\partial t}+u \frac{\partial}{\partial x}\right)^{2} \eta\right\}\left(1+\frac{\partial \eta}{\partial y}\right) \\
+\frac{\partial}{\partial y}\left\{\rho_{0}(y)\left(\frac{\partial}{\partial t}+u \frac{\partial}{\partial x}\right)^{2} \eta\right\} \frac{\partial \eta}{\partial x}=0
\end{gathered}
$$

and the second is,

$\frac{\partial u}{\partial x}\left(1+\eta_{y}\right)+\left(\frac{\partial}{\partial t}+\frac{\partial}{\partial x}\right) \eta_{y}=0$,

where

$N^{2}(y)=-\frac{1}{\sigma \rho_{0}(y)} \frac{d \rho_{0}}{d y}$.

The boundary conditions for these two equations are

$$
\begin{aligned}
& \frac{\partial \eta}{\partial x}=-\sigma\left(\frac{\partial u}{\partial t}+u \frac{\partial u}{\partial x}\right)-\left.\sigma \frac{\partial \eta}{\partial x}\left(\frac{\partial}{\partial t}+u \frac{\partial}{\partial x}\right)^{2} \eta\right|_{y=0} \\
& \eta=\left.0\right|_{y=-h} \cdot
\end{aligned}
$$

Thus, the basic equations in this semi-Lagrangian formulation are Eqs. (26) and (27) with boundary conditions at the surface (29) and bottom (30). We will use these to describe internal solitary waves. It is interesting to note that this semiLagrangian approach leads to an increase in the order of nonlinearity to four, whereas the original governing equations had only second order nonlinearity.

\section{Derivation of the nonlinear evolution equation}

We shall suppose that the waves are long, their amplitude is small, but finite, and that the basic horizontal shear flow is stable. We introduce the small parameter $\epsilon$ to describe long waves, and, hence, define the slow variables,

$X=\epsilon x, \quad T=\epsilon t$.

Then we decompose the horizontal velocity field into the basic component and a perturbation,

$u(x, y, t)=U(y)+u^{\prime}(x, y, t)$.

Equations (26) and (27) then transform to

$$
\begin{aligned}
& \frac{\partial}{\partial y}\left\{\rho_{0}(y)\left(\frac{\partial u^{\prime}}{\partial T}+\left(U(y)+u^{\prime}\right) \frac{\partial u^{\prime}}{\partial X}\right)\right\} \\
& -\rho_{0}(y) N^{2}(y) \frac{\partial \eta}{\partial X}-\epsilon^{2} \frac{\partial}{\partial X}\left\{\rho _ { 0 } ( y ) \left(\frac{\partial}{\partial T}\right.\right. \\
& \left.\left.+\left(U(y)+u^{\prime}\right) \frac{\partial}{\partial X}\right)^{2} \eta\right\} \cdot\left(1+\frac{\partial \eta}{\partial y}\right) \\
& +\epsilon^{2} \frac{\partial}{\partial y}\left\{\rho_{0}(y)\left(\frac{\partial}{\partial T}\right)\right. \\
& \left.\left.+\left(U(y)+u^{\prime}\right) \frac{\partial}{\partial X}\right)^{2} \eta\right\} \frac{\partial \eta}{\partial X}=0, \\
& \frac{\partial u^{\prime}}{\partial X}+\frac{\partial^{2} \eta}{\partial T \partial y}+\left(U(y)+u^{\prime}\right) \frac{\partial^{2} \eta}{\partial X \partial y}+\frac{\partial u^{\prime}}{\partial X} \frac{\partial \eta}{\partial y}=0 .
\end{aligned}
$$

The boundary condition at the surface (29) becomes

$$
\begin{aligned}
\frac{\partial \eta}{\partial X} & =-\sigma\left(\frac{\partial u^{\prime}}{\partial T}+\left(U(y)+u^{\prime}\right) \frac{\partial u^{\prime}}{\partial X}\right) \\
- & \sigma \epsilon^{2} \frac{\partial \eta}{\partial X}\left(\frac{\partial}{\partial T}+\left(U(y)+u^{\prime}\right) \frac{\partial}{\partial X}\right)^{2} \eta \\
\text { at } \quad y & =0,
\end{aligned}
$$

while the bottom boundary condition at the bottom (30) remains unchanged. We let the nonlinear parameter be $\mu$, and anticipate the $\mathrm{KdV}$ scaling $\mu=\epsilon^{2}$. If $c$ is the speed of a linear long wave (yet to be determined) we introduce the new variables,

$\xi=X-c T, \quad \tau_{1}=\mu T, \quad \tau_{2}=\mu^{2} T$,

It follows that

$$
\begin{aligned}
\frac{\partial}{\partial T} & =-c \frac{\partial}{\partial \xi}+\mu \frac{\partial}{\partial \tau_{1}}+\mu^{2} \frac{\partial}{\partial \tau_{2}}+\ldots, \\
\frac{\partial}{\partial X} & =\frac{\partial}{\partial \xi} .
\end{aligned}
$$

Then by substituting Eq. (37) into Eqs. (33), (34), and (35) we find that

$$
\frac{\partial}{\partial y}\left\{\rho_{0}(U-c) \frac{\partial u^{\prime}}{\partial \xi}\right\}-\rho_{0} N^{2} \frac{\partial \eta}{\partial \xi}=F,
$$




$$
\begin{aligned}
& \frac{\partial u^{\prime}}{\partial \xi}+(U-c) \frac{\partial^{2} \eta}{\partial \xi \partial y}=G \\
& \frac{\partial \eta}{\partial \xi}+\sigma(U-c) \frac{\partial u^{\prime}}{\partial \xi}= \\
& \quad-\left.\sigma\left(\mu \frac{\partial u^{\prime}}{\partial \tau_{1}}+\mu^{2} \frac{\partial u^{\prime}}{\partial \tau_{2}}+\ldots+u^{\prime} \frac{\partial u^{\prime}}{\partial \xi}+\mu \frac{\partial \eta}{\partial \xi} H\right)\right|_{y}
\end{aligned}
$$

where

$$
\begin{aligned}
F= & -\frac{\partial}{\partial y}\left\{\rho_{0}\left(\mu \frac{\partial u^{\prime}}{\partial \tau_{1}}+\mu^{2} \frac{\partial u^{\prime}}{\partial \tau_{2}}+\ldots+u^{\prime} \frac{\partial u^{\prime}}{\partial x}\right)\right\} \\
& +\mu\left(1+\frac{\partial \eta}{\partial y}\right) \frac{\partial}{\partial \xi}\left(\rho_{0} H\right)-\mu \frac{\partial \eta}{\partial \xi} \frac{\partial}{\partial y}\left(\rho_{0} H\right), \\
G= & -\mu \frac{\partial^{2} \eta}{\partial y \partial \tau_{1}}-\mu^{2} \frac{\partial^{2} \eta}{\partial y \partial \tau_{2}}+\ldots-\frac{\partial}{\partial \xi}\left(u^{\prime} \frac{\partial \eta}{\partial y}\right), \\
H= & \left((U-c) \frac{\partial}{\partial \xi}+\mu \frac{\partial}{\partial \tau_{1}}+\mu^{2} \frac{\partial}{\partial \tau_{2}}+\ldots\right. \\
& \left.+u^{\prime} \frac{\partial}{\partial \xi}\right)^{2} \eta .
\end{aligned}
$$

Here the left-hand side of these equations, when equated to zero, describe the linear long-wave theory, and thus, form the basis of our asymptotic expansion.

Equations (38) and (39) can be reduced to one equation containing $\eta$ only,

$$
\frac{\partial}{\partial y}\left\{\rho_{0}(U-c)^{2} \frac{\partial^{2} \eta}{\partial \xi \partial y}\right\}+\rho_{0} N^{2} \frac{\partial \eta}{\partial \xi}=M,
$$

where

$M=\frac{\partial}{\partial y}\left\{\rho_{0}(U-c) G\right\}-F$

with the boundary conditions,

$$
\frac{\partial \eta}{\partial \xi}=\sigma(U-c)^{2} \frac{\partial^{2} \eta}{\partial \xi \partial y}-\sigma(U-c) G+\left.\sigma H_{1}\right|_{y=0}
$$

where

$$
H_{1}=-\left(\mu \frac{\partial u^{\prime}}{\partial \tau_{1}}+\mu^{2} \frac{\partial u^{\prime}}{\partial \tau_{2}}+\ldots+u^{\prime} \frac{\partial u^{\prime}}{\partial \xi}+\mu \frac{\partial \eta}{\partial \xi} H\right)
$$

Again Eq. (30) holds at the bottom.

Next we assume that our internal wave field (i.e. the vertical displacement and the horizontal component of velocity) has the asymptotic expansion,

$$
\begin{aligned}
& \eta(\xi, y, \boldsymbol{\tau})=\mu A(\xi, \boldsymbol{\tau}) \Phi(y)+\mu^{2} \eta_{1}(\xi, y, \boldsymbol{\tau}) \\
& \quad+\mu^{3} \eta_{2}(\xi, y, \boldsymbol{\tau})+\ldots \\
& u^{\prime}(\xi, y, \boldsymbol{\tau})=\mu u_{0}(\xi, y, \boldsymbol{\tau})+\mu^{2} u_{1}(\xi, y, \boldsymbol{\tau}) \\
& \quad+\mu^{3} u_{2}(\xi, y, \boldsymbol{\tau})+\ldots
\end{aligned}
$$

Here $\boldsymbol{\tau}$ is a vector representing the multiple time scales $\left(\tau_{1}, \tau_{2}, \ldots\right)$. After substitution of Eqs. (48) and (49) into
Eq. (44) and the boundary conditions (30) and (46) and collecting terms of the same order in $\mu$, we obtain at the lowest order the equation determining the modal function $\Phi(y)$,

$$
\begin{aligned}
& \frac{d}{d y}\left[\rho_{0}(c-U)^{2} \frac{d \Phi}{d y}\right]+\rho_{0} N^{2} \Phi=0 \\
& \Phi=\left.0\right|_{y=h} \\
& \Phi=\left.\sigma(c-U)^{2} \frac{d \Phi}{d y}\right|_{y=0} .
\end{aligned}
$$

It is well-known that this eigenvalue problem (50), (51) and (52) has, in general, an infinite sequence of modes $\Phi_{n}^{ \pm}$with corresponding speeds $c_{n}^{ \pm}$, for $n=0,1,2, \ldots$ Here we consider only stable waves, so that $c_{n}^{+}>U_{M}=\max U(y)$ and $c_{n}^{-}<U_{m}=\min U(y)$. Note that a sufficient condition to exclude any unstable waves (where $U_{m}<\operatorname{Re}(c)<U_{M}$ ) is that the Richardson number $R i=N^{2} / U_{y}^{2}>1 / 4$. The theory we shall develop is valid for any of these modes, but usually it is relevant to consider only the first internal mode, which has the greatest phase speed of all the internal modes and has just a single extremum for the modal function in the interior of the fluid. Also, it is important to note that each mode is determined only to within a multiplicative constant. We choose this constant in such a way that modal function is normalized at its extreme value, i.e.

$\Phi_{\max }=1$.

This choice of modal function has an obvious physical sense, in that at the first order of approximation, the function $A(\xi, \tau)$ is the displacement of the isopycnal surface at the point where $\Phi=\Phi_{\max }$, denoted by $y=y_{\max }$.

From Eq. (39), substituting Eqs. (48) and (49), at the lowest order we find

$u_{0}(\xi, y, \boldsymbol{\tau})=-A(\xi, \boldsymbol{\tau})(U-c) \frac{d \Phi}{d y}$.

Equation (44) can be written in operator form

$L \frac{\partial \eta}{\partial \xi}=M$.

Here $L$ is the linear operator,

$L=\frac{\partial}{\partial y}\left[\rho_{0}(c-U)^{2} \frac{\partial}{\partial y}\right]+\rho_{0} N^{2}$.

The compatibility condition, i.e. the condition for solvability of the inhomogeneous problem (55) with the boundary conditions (30) and (46), is

$\int_{-h}^{0} M \Phi d y=\sigma\left[\rho_{0}(U-c)^{2} \frac{d \Phi}{d y}\left((U-c) G-H_{1}\right)\right]_{y=0}$

or, using Eq. (45):

$$
\begin{aligned}
\int_{-h}^{0} F \Phi d y+\int_{-h}^{0} \rho_{0}(U-c) G \frac{d \Phi}{d y} d y \\
-\sigma\left[\rho_{0}(U-c)^{2} H_{1} \frac{d \Phi}{d y}\right]_{y=0}=0 .
\end{aligned}
$$


It is useful to note that

$F=\frac{\partial F_{1}}{\partial y}+\frac{\partial F_{2}}{\partial \xi}$,

where, from Eqs. (41) and (47):

$F_{1}=\rho_{0} H_{1}$

$F_{2}=\mu \rho_{0}\left(1+\frac{\partial \eta}{\partial y}\right) H$.

Hence, the compatibility condition (57) now becomes:

$$
\begin{aligned}
\int_{-h}^{0} & \frac{\partial F_{2}}{\partial \xi} \Phi d y-\int_{-h}^{0} F_{1} \frac{d \Phi}{d y} d y \\
& +\int_{-h}^{0} \rho_{0}(U-c) G \frac{d \Phi}{d y} d y=0,
\end{aligned}
$$

and so it does not contain the boundary terms.

We'll consider the expression (61) at each order of $\mu$. At $O\left(\mu^{2}\right)$ we obtain:

$\frac{\partial A}{\partial \tau_{1}}+\beta \frac{\partial^{3} A}{\partial \xi^{3}}+\alpha A \frac{\partial A}{\partial \xi}=0$

where

$\alpha=\frac{3}{2} \frac{\int_{-h}^{0} \rho_{0}(c-U)^{2}(d \Phi / d y)^{3} d y}{\int_{-h}^{0} \rho_{0}(c-U)(d \Phi / d y)^{2} d y}$

$\beta=\frac{1}{2} \frac{\int_{-h}^{0} \rho_{0}(c-U)^{2} \Phi^{2} d y}{\int_{-h}^{0} \rho_{0}(c-U)(d \Phi / d y)^{2} d y}$.

Equation (62) is the well-known $\mathrm{KdV}$ equation for internal solitary waves. Note that Eq. (62) is expressed in a moving coordinate system, and when Eq. (37) is used we obtain the same equation but in the fixed coordinate system,

$\frac{\partial A}{\partial T}+c \frac{\partial A}{\partial X}+\mu\left(\beta \frac{\partial^{3} A}{\partial X^{3}}+\alpha A \frac{\partial A}{\partial X}\right)=0$

The coefficients $\alpha$ and $\beta$ of this $\mathrm{KdV}$ equation were first obtained by Benney (1966).

Equation (44) at $O\left(\mu^{2}\right)$ is

$$
\begin{aligned}
L \frac{\partial \eta_{1}}{\partial \xi}=\frac{\partial^{3} A}{\partial \xi^{3}}\left[-2 \beta \frac{\partial}{\partial y}\left\{\rho_{0}(c-U) \frac{d \Phi}{d y}\right\}\right. & \left.-\rho_{0}(c-U)^{2} \Phi\right] \\
+A \frac{\partial A}{\partial \xi}\left[-2 \alpha \frac{\partial}{\partial y}\left\{\rho_{0}(c-U) \frac{d \Phi}{d y}\right\}\right. & \left.+3 \frac{\partial}{\partial y}\left\{\rho_{0}(c-U)^{2}\left(\frac{d \Phi}{d y}\right)^{2}\right\}\right]
\end{aligned}
$$

Therefore,

$\eta_{1}=\frac{\partial^{2} A}{\partial \xi^{2}} T_{d}(y)+A^{2} T_{n}(y)$,

where $T_{n}(y)$ is the first nonlinear correction to the modal structure of internal wave; it is the solution of

$$
\begin{aligned}
L T_{n} & =-\alpha \frac{d}{d y}\left\{\rho_{0}(c-U) \frac{d \Phi}{d y}\right\} \\
& +\frac{3}{2} \frac{d}{d y}\left\{\rho_{0}(c-U)^{2}\left(\frac{d \Phi}{d y}\right)^{2}\right\}
\end{aligned}
$$

with boundary conditions

$$
\begin{aligned}
T_{n}= & \left.0\right|_{y=-h}, \\
T_{n}= & \sigma\left[(c-U)^{2} \frac{d T_{n}}{d y}+\alpha(c-U) \frac{d \Phi}{d y}\right. \\
& \left.+\frac{3}{2}(c-U)^{2}\left(\frac{d \Phi}{d y}\right)^{2}\right]\left.\right|_{y=0},
\end{aligned}
$$

while $T_{d}(y)$ is the first dispersion correction to the modal structure of internal wave; it is solution of

$L T_{d}=-2 \beta \frac{d}{d y}\left\{\rho_{0}(c-U) \frac{d \Phi}{d y}\right\}-\rho_{0}(c-U)^{2} \Phi$

with boundary conditions

$$
\begin{aligned}
& T_{d}=\left.0\right|_{y=-h}, \\
& T_{d}=\left.\sigma\left[(c-U)^{2} \frac{d T_{d}}{d y}+2 \beta(c-U) \frac{d \Phi}{d y}\right]\right|_{y=0} .
\end{aligned}
$$

It is important to note that solutions of the boundary-value problems (68), (69) and (70), (71) are unique only up to additive multiples of $\Phi$. This problem was discussed in Lamb and Yan (1996), Lamb (1999) and Holloway et al. (1999). It is convenient to let $A\left(\xi, \tau_{1}\right)$ represent the isopycnal displacement at the level $y_{\max }$ where there is a maximum in the linear mode $\Phi(y)$. Hence, we choose the auxiliary conditions

$T_{n}\left(y_{\max }\right)=0, \quad T_{d}\left(y_{\max }\right)=0$.

In this case the series (48), using Eqs. (67) and (53), at the point $y_{\max }$ is

$\eta\left(\xi, y_{\max }, \tau_{1}\right)=\mu A\left(\xi, \tau_{1}\right)+O\left(\mu^{3}\right)$.

Also, Eqs. (39) at $O\left(\mu^{2}\right)$ and (67) give

$$
\begin{aligned}
u_{1}= & \frac{\partial^{2} A}{\partial \xi^{2}}\left[\beta \frac{d \Phi}{d y}-(U-c) \frac{d T_{d}}{d y}\right]+A^{2} \\
& \cdot\left[\frac{1}{2} \alpha \frac{d \Phi}{d y}+(U-c)^{2}\left(\frac{d \Phi}{d y}\right)^{2}-(U-c) \frac{d T_{n}}{d y}\right] .
\end{aligned}
$$

The compatibility condition $(61)$ at $O\left(\mu^{3}\right)$ then generates the equation,

$$
\begin{aligned}
\frac{\partial A}{\partial \tau_{2}} & +\beta_{1} \frac{\partial^{5} A}{\partial \xi^{5}}+\alpha_{1} A^{2} \frac{\partial A}{\partial \xi}+\gamma_{1} A \frac{\partial^{3} A}{\partial \xi^{3}} \\
& +\gamma_{2} \frac{\partial A}{\partial \xi} \frac{\partial^{2} A}{\partial \xi^{2}}=0,
\end{aligned}
$$


where

$$
\begin{aligned}
& \beta_{1}=\frac{1}{I} \int_{-h}^{0} \rho d y\left\{(c-U)^{2} \Phi T_{d}-\beta^{2}\left(\frac{d \Phi}{d y}\right)^{2}\right. \\
& \left.+2 \beta(c-U)\left[\Phi^{2}-\left(\frac{d \Phi}{d y}\right)\left(\frac{d T_{d}}{d y}\right)\right]\right\}, \\
& \alpha_{1}=\frac{1}{I} \int_{-h}^{0} \rho d y\left\{3(c-U)^{2}\left[3\left(\frac{d T_{n}}{d y}\right)-2\left(\frac{d \Phi}{d y}\right)^{2}\right]\right. \\
& \cdot\left(\frac{d \Phi}{d y}\right)^{2}-\alpha^{2}\left(\frac{d \Phi}{d y}\right)^{2}+\alpha(c-U) \\
& \left.\cdot\left[5\left(\frac{d \Phi}{d y}\right)^{2}-4\left(d T_{n} / d y\right)\right]\left(\frac{d \Phi}{d y}\right)\right\}, \\
& \gamma_{1}=-\frac{1}{I} \int_{-h}^{0} \rho d y\left\{2 \alpha \beta\left(\frac{d \Phi}{d y}\right)^{2}-2 \alpha(c-U) \Phi^{2}\right. \\
& +(c-U)^{2} \Phi^{2}\left(\frac{d \Phi}{d y}\right)-(c-U)^{2}\left[2 T_{n} \Phi\right. \\
& \left.+3\left(\frac{d T_{d}}{d y}\right)\left(\frac{d \Phi}{d y}\right)^{2}\right]+2(c-U)\left[\alpha\left(\frac{d T_{d}}{d y}\right)\right. \\
& \left.\left.+2 \beta\left(d T_{n} / d y\right)\right]\left(\frac{d \Phi}{d y}\right)-4 \beta(c-U)\left(\frac{d \Phi}{d y}\right)^{3}\right\}, \\
& \gamma_{2}=\frac{1}{I} \int_{-h}^{0} \rho d y\left\{(c-U)\left[2 \beta\left(\frac{d \Phi}{d y}\right)^{3}+6 \alpha \Phi^{2}\right]\right. \\
& -3 \alpha \beta\left(\frac{d \Phi}{d y}\right)^{2}-2(c-U)^{2}\left[\Phi^{2}\left(\frac{d \Phi}{d y}\right)-3 T_{n} \Phi\right] \\
& -6 \alpha(c-U)\left(\frac{d T_{d}}{d y}\right)\left(\frac{d \Phi}{d y}\right) \\
& \left.+3(c-U)^{2}\left(\frac{d T_{d}}{d y}\right)\left(\frac{d \Phi}{d y}\right)^{2}\right\}, \\
& I=2 \int_{-h}^{0} \rho(c-U)(d \Phi / d y)^{2} d y .
\end{aligned}
$$

Then, again using Eq. (37), and the KdV equation (62), and neglecting terms of $O\left(\mu^{3}\right)$ we obtain the second-order $\mathrm{KdV}$ equation, or extended $\mathrm{KdV}$ equation,

$$
\begin{aligned}
\frac{\partial A}{\partial T} & +c \frac{\partial A}{\partial X}+\mu\left(\beta \frac{\partial^{3} A}{\partial X^{3}}+\alpha A \frac{\partial A}{\partial X}\right)+\mu^{2}\left(\beta_{1} \frac{\partial^{5} A}{\partial X^{5}}\right. \\
& \left.+\alpha_{1} A^{2} \frac{\partial A}{\partial X}+\gamma_{1} A \frac{\partial^{3} A}{\partial X^{3}}+\gamma_{2} \frac{\partial A}{\partial X} \frac{\partial^{2} A}{\partial X^{2}}\right)=0 .
\end{aligned}
$$

This equation was produced by Koop and Butler (1981) for a two-layer system, and then by Lamb and Yan (1996) for a continuous density stratification, but in the Boussinesq approximation, with no free surface and without a basic shear flow. Recently, this last result was extended by Pelinovsky, Poloukhina, Lamb (2000) to include a basic shear flow, but again in the Boussinesq approximation and with no free surface. It is important to note that our derivation is completely general, and in particular, also includes the case of a surface wave, in which case one should choose $y_{\max }=0$. Further, the procedure described here allows us, in principle, to obtain the extension of the Korteweg-de Vries equation to any order. However, as shown by Prasad and Akylas (1997) for the case when the upper boundary is rigid and there is no basic shear flow, we would expect the generation of both upstream and downstream shelves at $O\left(\mu^{4}\right)$, which are associated with the necessity for the total asymptotic expansion to conserve mass.

\section{Interfacial waves in a two-layer shear flow}

Consider a two-layer system, bounded below by a rigid flat bottom and above by a free surface. The lower and upper layer densities are $\rho_{1}$ and $\rho_{2}\left(\rho_{1}>\rho_{2}\right)$, and the corresponding layer depths are $h$ and $H-h$, i.e. $H$ is the undisturbed fluid depth; note the change of notation from the general theory of the preceding sections. This case was analyzed by Koop and Butler (1981) without any shear flow. Here we include a shear flow by including a constant velocity $U_{0}$ in the upper layer.

The parameter $\sigma$ (Boussinesq parameter) for this twolayer case is

$\sigma=2 \frac{\rho_{1}-\rho_{2}}{\rho_{1}+\rho_{2}}$.

The vertical structure of the modal function corresponding to the internal mode can be found from the eigenvalue problem (50), (51) and (52), and has the form

$\Phi(y)= \begin{cases}y / h, & 0 \leq y<h, \\ (y+m-h) / m, & h \leq y \leq H,\end{cases}$

where

$m=\sigma\left(c-U_{0}\right)^{2}+h-H$.

Note that the fluid domain is now given by $0<y<H$, but all the formulae of the preceding sections are readily altered accordingly. The linear long-wave phase speed $c$ is a solution of

$$
\begin{array}{r}
-c^{2}\left(c-U_{0}\right)^{2} \sigma(2+\sigma)+c^{2}(2+\sigma)(H-h)+ \\
+\left(c-U_{0}\right)^{2} h(2+\sigma)-2 h(H-h)=0 .
\end{array}
$$

The phase speed can be expressed in semi-explicit form

$$
c^{2}=\frac{(2+\sigma)\left(H-h+(1-u)^{2} h\right)-\sqrt{D}}{2(1-u)^{2} \sigma(2+\sigma)},
$$

where

$$
\begin{aligned}
D= & (2+\sigma)\left[(2+\sigma)\left(H-h+(1-u)^{2} h\right)^{2}\right. \\
& \left.-8(1-u)^{2} \sigma h(H-h)\right],
\end{aligned}
$$

and $u=U_{0} / c$ is the relative shear flow velocity. An increase in $\sigma$ causes a decrease in the phase speed $c$. 
From Eqs. (68)-(72) we can find the nonlinear and dispersion corrections to the modal function (83). The nonlinear correction is given by,

$$
T_{n}(y)= \begin{cases}0, & 0 \leq y<h \\ a_{1}(h-y), & h \leq y \leq H\end{cases}
$$

where

$a_{1}=\frac{m+H-h}{m^{2}\left(c-U_{0}\right)}\left(\alpha-\frac{3}{2} \frac{\left(c-U_{0}\right)}{m}\right)$,

while the dispersion correction has the form,

$$
T_{d}(y)=\left\{\begin{array}{rr}
-\frac{y^{3}}{6 h}+\frac{h y}{6}, & 0 \leq y<h \\
-\frac{(y-h+m)^{3}}{6 m}+a_{2}(y-h)+ & \\
+\frac{m^{2}}{6}, & h \leq y \leq H
\end{array}\right.
$$

where

$a_{2}=-\frac{2 \beta(m+H-h)}{\left(c-U_{0}\right) m^{2}}+\frac{(m+H-h)^{3}}{3 m^{2}}+\frac{m}{6}$.

Next, from Eqs. (63), (64), (76), (77), (78), and (79) we can evaluate the coefficients of the extended Korteweg - de Vries Eq. (81) written here for the interfacial vertical displacement. Formulas for these quantities are complicated and, hence, are given in Appendix A. First, we note that in the case of the Boussinesq approximation, when the density jump is small (i.e. $\sigma \approx 0$, or $\rho_{1} \approx \rho_{2}$ ), so that the influence of the free surface is then also small and upper boundary is effectively rigid, all coefficients coincide with those calculated by Pelinovsky, Poloukhina, Lamb (2000). Hence, our emphasis here is to explore, inter alia, how these coefficients may vary with $\sigma$, given here by Eq. (82).

In Figs. 1-3 we display graphs of the non-dimensionalised quantities $\alpha, \beta, \alpha_{1}, \beta_{1}, \gamma_{1}, \gamma_{2}$ as functions of $l=h / H$ for different values of the relative shear flow velocity $u=U_{0} / c$, and the relative density $r=\rho_{1} / \rho_{2}$. Note that as $l$ and $r$ vary, so does $c$ and, hence, so does $U_{0}$ when we keep $u$ fixed.

First, we note that the nonlinear coefficients $\alpha$ and $\alpha_{1}$ have infinite values when $l=0, l=1$, i.e. when the thickness of either the lower layer, or that of the upper layer, tends to zero. The coefficient $\gamma_{2}$ of the nonlinear dispersion term also has an infinite value at $l=1$, when $r>1$. Of course, our results are not valid in the vicinity of the such points.

The coefficient $\alpha$ of the quadratic nonlinear term is positive when the pycnocline near the bottom and negative when pycnocline is near to the surface. For $u=0$ and $\sigma \rightarrow 0$ $\alpha>0(<0)$ according as $h<(>) H / 2$. The effect of increasing the shear flow velocity is to put nearer to the surface the location where $\alpha$ is equal to zero. Varying the relative density $r$ has a very weak effect in the region of positive $\alpha$, but its effect is more noticeable in the region where $\alpha$ is negative; increasing $r$ causes an increase in the absolute value of $\alpha$.

The coefficient $\alpha_{1}$ of the cubic nonlinear term is negative for any layer depth ratio, and takes smaller (in modulus) values as the relative shear flow velocity $u$ increases. When the lower layer is thin enough, the density ratio $r$ has almost no influence on the cubic nonlinear coefficient, but when the thickness of the lower layer is not so small, we obtain larger absolute values of $\alpha_{1}$ for larger values of $r$.

The coefficient $\beta$ of the first-order dispersion term is zero at $l=0$ and $l=1$ and positive for any other layer depth ratio, and, hence, it has a maximum value whose magnitude and location depend on the values of the parameters $u$ and $r$. When there is no shear flow $(u=0), \beta(l)$ is symmetric function, and its maximum is at $l=1 / 2$ (i.e. equal layer depths), while the effect of increasing $r$ is to decrease the maximum of $\beta$. The presence of a shear flow destroys the symmetry of $\beta(l)$, and the position of its maximum moves to larger $l$ with an increase in $u$.

The coefficient $\beta_{1}$ of the second order dispersion term has a behaviour similar to that of $\beta$ for $r$ close to 1 . However, when the difference in layer densities is significant, $\beta_{1}$ can take negative values if the upper layer is thinner than the lower. The qualitative behaviour of the coefficients $\gamma_{1,2}$ of the nonlinear dispersion terms is similar to that of $\alpha$, except when the pycnocline is near to the bottom (when $\gamma_{1}$ and $\gamma_{2}$ take finite positive values), or when the pycnocline is near to the surface (when $\gamma_{1}$ has finite negative values, and $\gamma_{2}$ has infinite positive values for $r>1$ and finite negative values for $r=1)$.

Since the basic state considered here has a jump in the basic current field across the density-interface, this model is, in principle, subject to high-wave number Kelvin-Helmholtz instability. However, because we are dealing here with long waves, this issue need not necessarily cause too much concern, and indeed, it is quite common to regard such layered models as valid approximations to continuous basic density and current profiles when, as here, one is considering long waves. In order to demonstrate this explicitly in the present context, we replaced the two-layer model considered here with a three-layer model, in which the lower layer has a constant density $\rho_{1}$, and the upper layer has a constant density $\rho_{2}$ and a constant current $U_{0}$. The middle layer is thin, with a thickness $\delta$, and has a constant bouyancy frequency $N_{0}$ and a constant current shear $U_{0} / \delta$, both constructed to ensure that the basic density and current profiles are everywhere continuous. The details are given in Appendix B. For this case the coefficients $\alpha, \beta, \alpha_{1}, \beta_{1}, \gamma_{1}$ and $\gamma_{2}$ were evaluated numerically. A particular case when $\delta=H / 100$ and when the Richardson number in the thin shear layer was 0.55 (so that this basic profile is linearly stable) is shown in Fig. 4, and compared with the corresponding results for the two-layer model considered above. For all coefficients there is good agreement.

Although our primary purpose here is to consider internal solitary waves, we recall that all the formulas obtained in Sect. 4 are also valid for the surface wave mode. Hence, in Appendix C, we present the results for the coefficients $\alpha, \beta, \alpha_{1}, \beta_{1}, \gamma_{1}$ and $\gamma_{2}$ for the surface wave mode of the twolayer model introduced at the beginning of this section. In particular, we can the verify that these expressions reduce to the well-known ones for surface solitary waves when the two-layer model is collapsed into a single layer. 

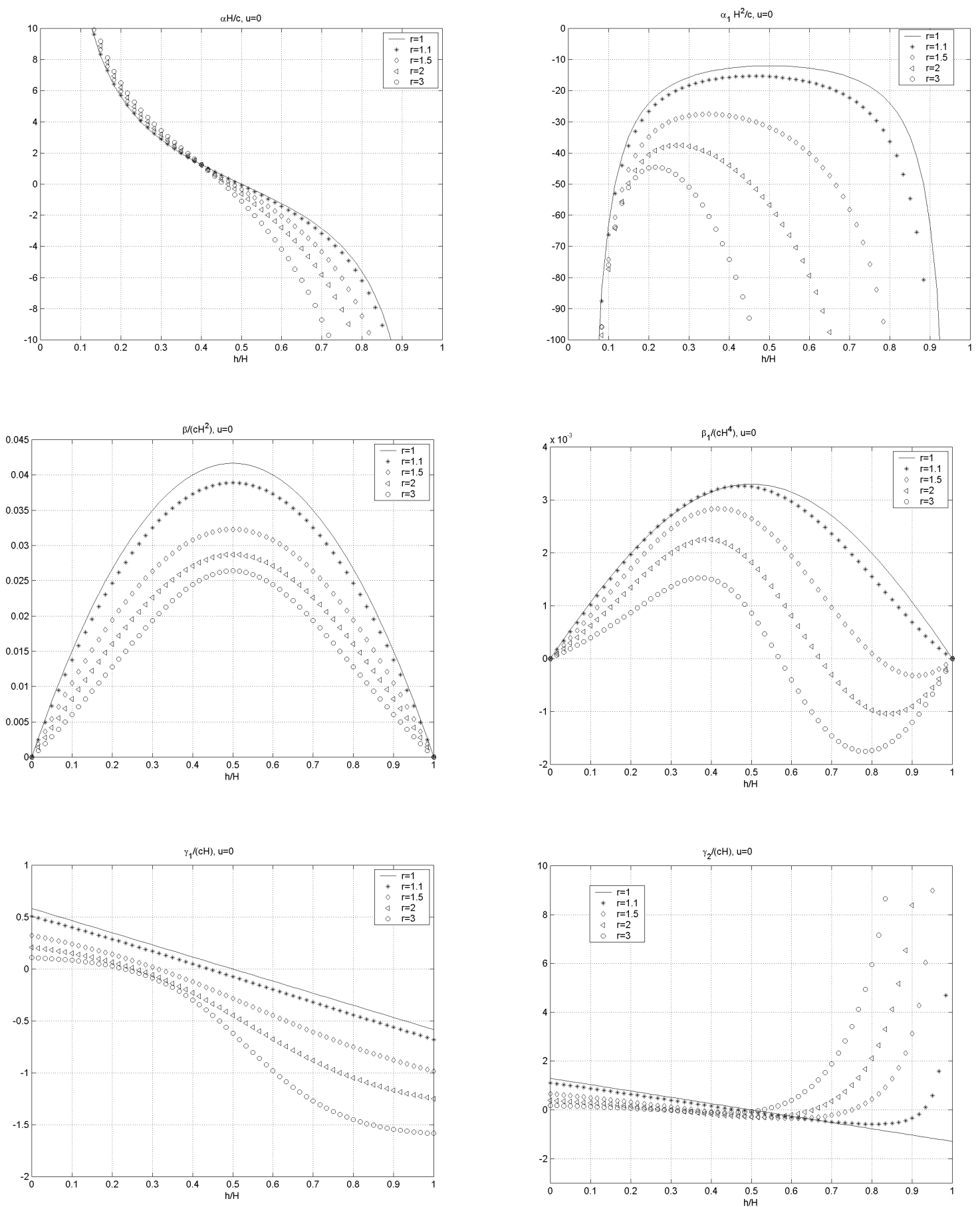

Fig. 1. Coefficients of the extended Korteweg-de Vries equation for a two-layer fluid with no current.

\section{Conclusion}

We have presented an evolution equation, the extended Korteweg de-Vries equation, to describe solitary waves in an arbitrary density- and current-stratified flow, without using the Boussinesq approximation and with a free surface, valid to the second order of perturbation theory. All the coefficients of this equation are given explicitly as integrals of the modal function, together with its nonlinear and dispersion correc- tions.

The special case of a two-layer fluid with a shear flow due to a constant velocity in the upper layer is discussed in detail. For this special case the coefficients are obtained explicitly in terms of the parameters of the model (layer depths, density ratio and the relative shear flow velocity), and are analysed as functions of these parameters. It is shown that the influence of the density ratio, and the shear flow velocity, is significant for all the coefficients, and should not be neglected in 

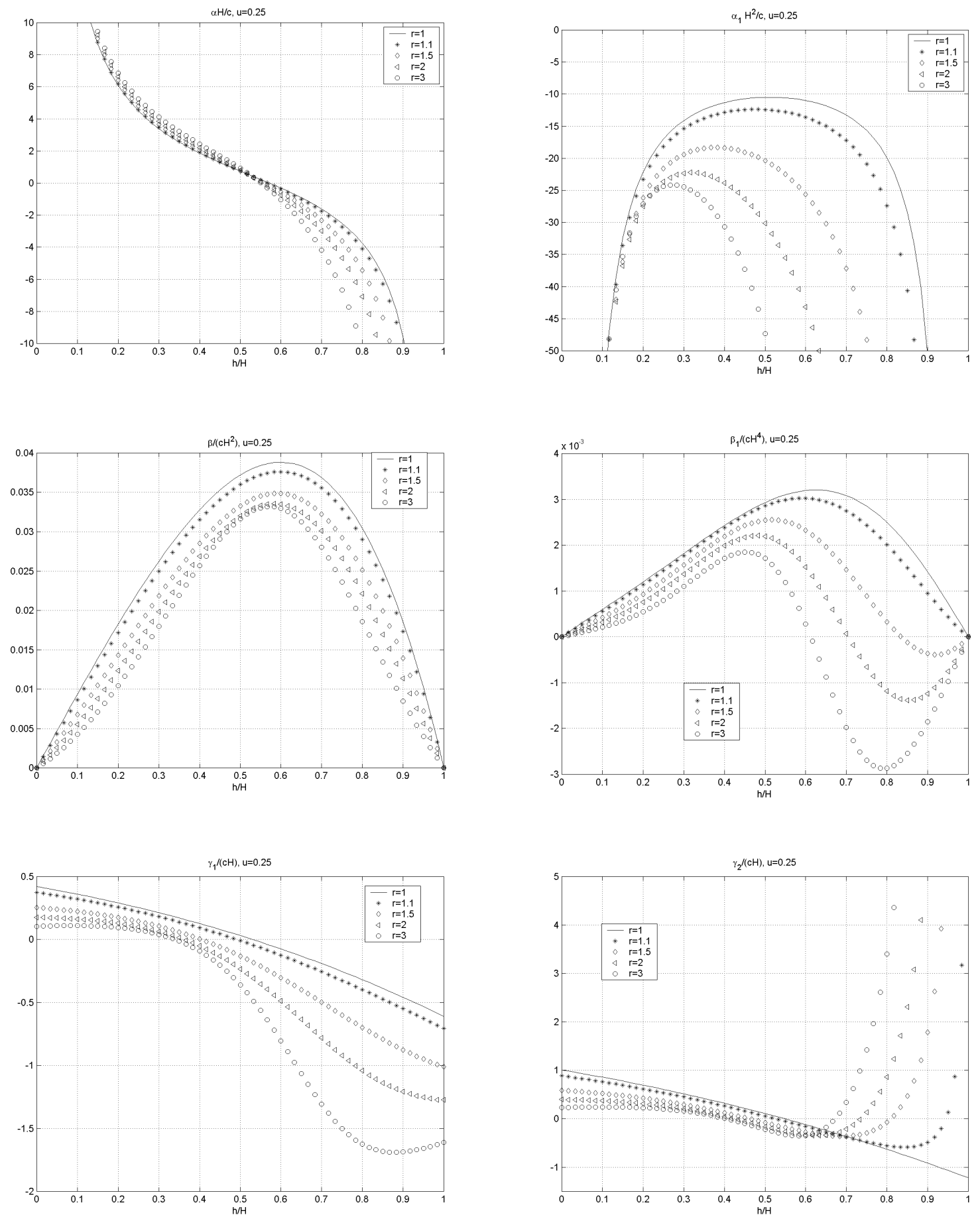

Fig. 2. Coefficients of the extended Korteweg-de Vries equation for a two-layer fluid with a current in upper layer, $\left(u=U_{0} / c=0.25\right)$.

general.

Next, we must point out again that the higher-order Korteweg-de Vries equation (81) is, strictly speaking, an asymptotic result valid when $\mu$ is sufficiently small, and is most likely to be useful when the coefficient $\alpha$ of the quadratic nonlinear term is small (e.g. $O(\epsilon)$ where we recall that $\mu=\epsilon^{2}$ ). However, because observed internal solitary waves are often quite large, we suggest that in practice it may be useful to use Eq. (81) as the model equation even when $\mu$ is not small.

In conclusion, we note that although the higher-order Korteweg-de Vries equation (81), and the expressions defining the associated nonlinear and dispersion corrections (68) and (70) to the modal functions, are all uniquely defined with our choice of normalisation (see Eqs. 53 and 72), a different choice for the normalisation would produce a different 

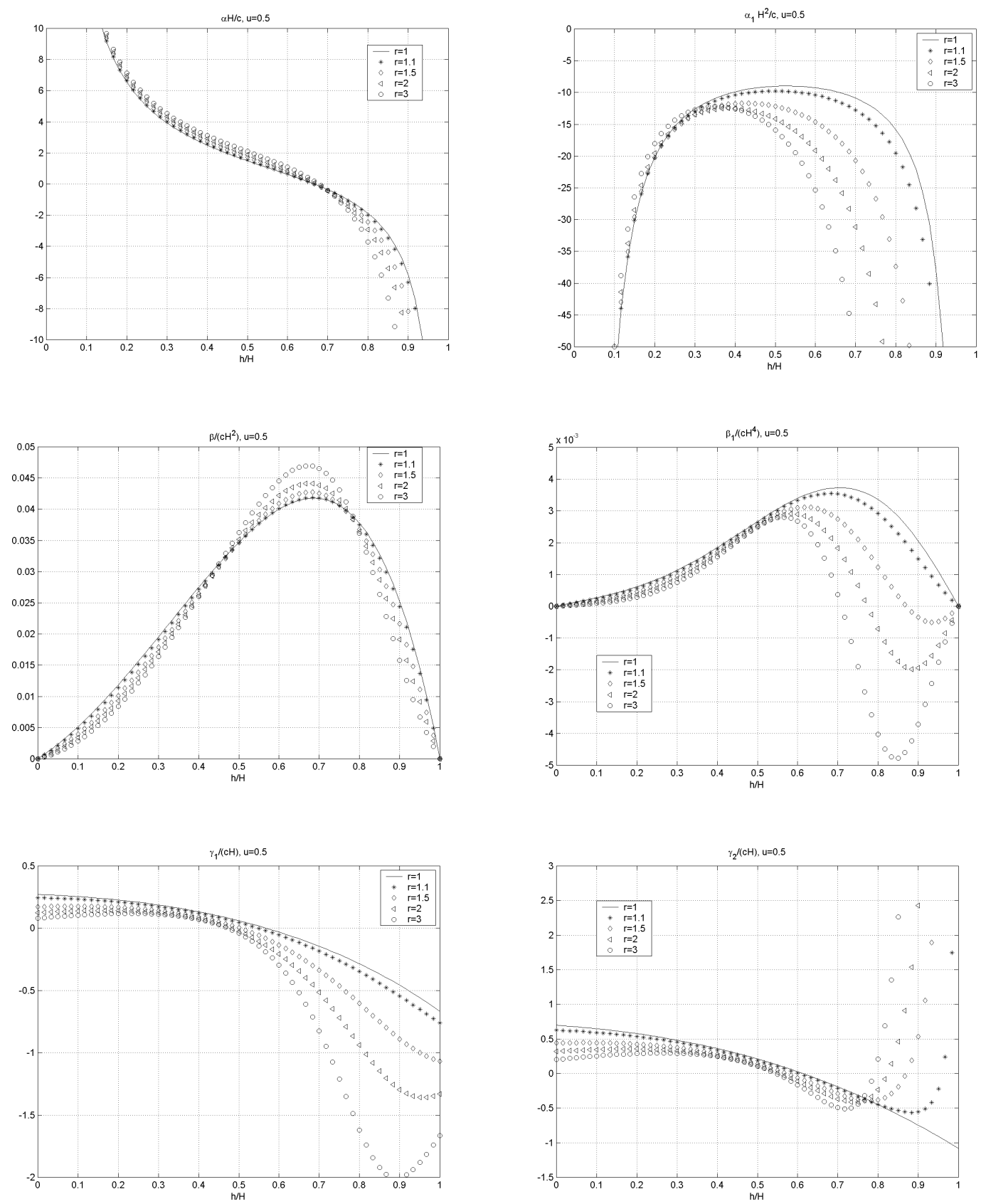

Fig. 3. Coefficients of the extended Korteweg-de Vries equation for a two-layer fluid with a current in upper layer $\left(u=U_{0} / c=0.5\right)$.

set of coefficients. In particular, we recall that the nonlinear and dispersion corrections to the modal function, $T_{n}(y)$ and $T_{d}(y)$, respectively, are only defined by Eqs. (68) and (70) to within an arbitrary multiple of the modal function $\Phi(y)$ itself; it is only the normalisation conditions (72) which then uniquely determine them. Omitting these normalisation conditions has the effect of allowing us to make a near-identity transformation

$B=A+\mu\left\{\frac{1}{2} a A^{2}+b A_{X X}\right\}$

where a specific choice of the coefficients $a, b$ represents a specific normalisation of $T_{n}(y), T_{d}(y)$, respectively. It can now be readily verified that substitution of Eq. (89) into Eq. (81) allows us to generate asymptotically a higherorder Korteweg-de Vries equation for $B$ of the same form as Eq. (81), but with the coefficients $\beta_{1}, \alpha_{1}, \gamma_{1}$ and $\gamma_{2}$ replaced by $\beta_{1}, \alpha_{1}-a \alpha / 2, \gamma_{1}$ and $\gamma_{2}-3 a \beta+2 b \alpha$, respectively. Note that the coefficients $\alpha, \beta$ in the first-order Korteweg-de Vries equation are not changed, nor are the coefficients $\beta_{1}$ and $\gamma_{1}$ of the higher-order dispersive term. Indeed, it is easily seen from the integral expressions (76) and (78) for $\beta_{1}$ and $\gamma_{1}$, respectively, that adding a multiple of $\phi$ to $T_{d}$ and/or $T_{n}$ leaves $\beta_{1}$ and $\gamma_{1}$ unchanged.

In particular, we can now choose $a$ so that the higher- 

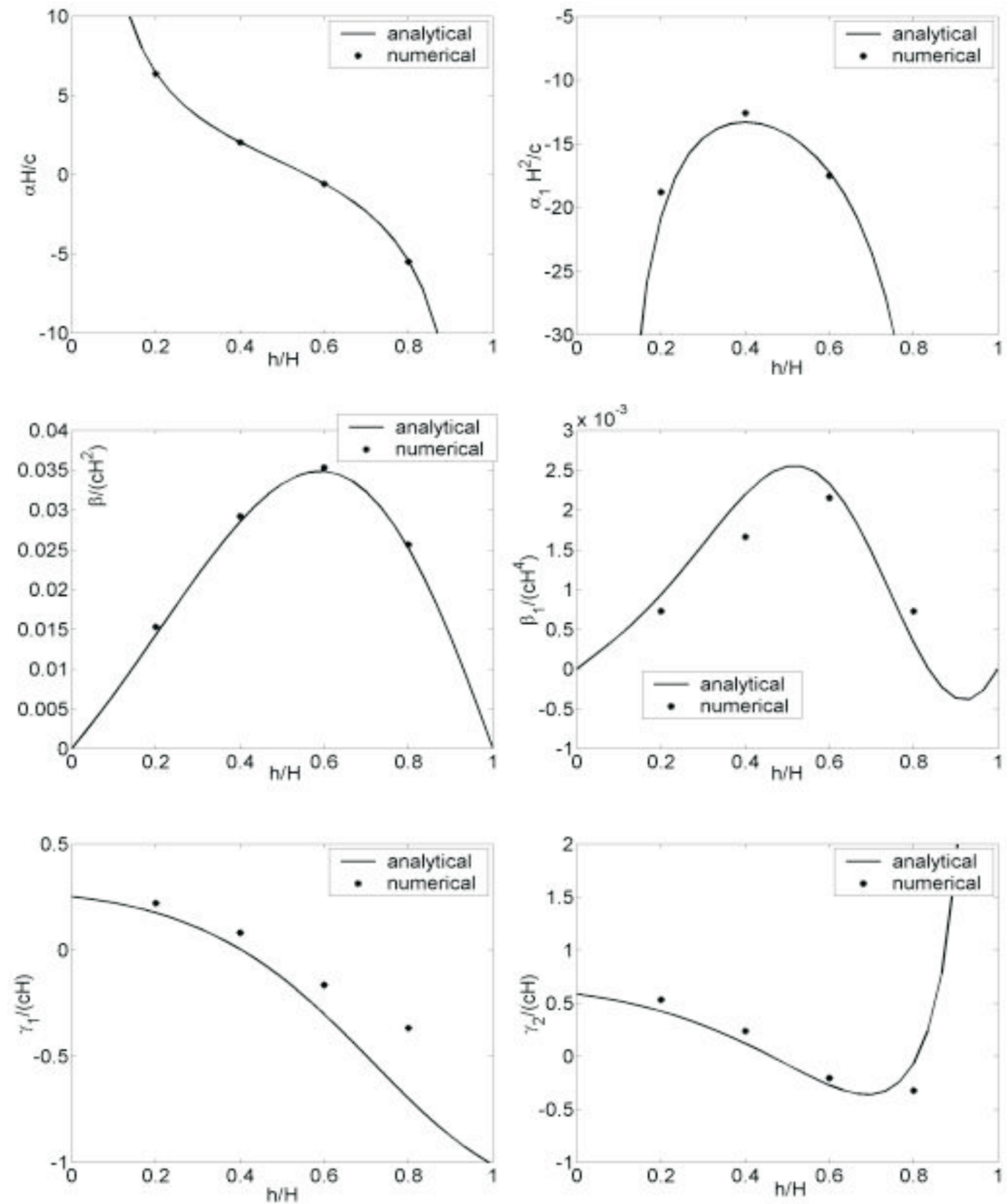

Fig. 4. Numerical calculation of the coefficients of the extended Korteweg-de Vries equation for a three-layer flow with a thin intermediate layer. The solid line corresponds to the analytical expressions for the corresponding two-layer model.

order Korteweg-de Vries equation for $B$ is Hamiltonian. For Eq. (81) to be exactly Hamiltonian (as opposed to being only asymptotically Hamiltonian), it is necessary that $\gamma_{2}=2 \gamma_{1}$, which is generally not the case (see Eqs. 78 and 79). However, the near-identity transformation (89) with the choice $3 a \beta=\gamma_{2}-2 \gamma_{1}$ and $b=0$ will produce a Hamiltonian form. With $\gamma_{2}=2 \gamma_{1}$ the Hamiltonian form for Eq. (81) is

$A_{T}=-\frac{\partial}{\partial X} \frac{\delta H}{\delta A}$ where the Hamiltonian $H$ has the density

$$
\begin{aligned}
\frac{1}{2} c A^{2} & +\mu\left(-\frac{1}{2} \beta A_{X}^{2}+\frac{1}{6} \alpha A^{3}\right) \\
& +\mu^{2}\left(\frac{1}{2} \beta_{1} A_{X X}^{2}+\frac{1}{12} \alpha_{1} A^{4}-\frac{1}{2} \gamma_{1} A A_{X}^{2}\right) .
\end{aligned}
$$

When Eq. (81) is Hamiltonian then it conserves not only the 
mass (i.e. the integral of $A$ ), but also the momentum whose density is $A^{2}$, as well as the Hamiltonian itself, whose density is $H$. For numerical purposes it is perhaps desirable that the evolution Eq. (81) should be Hamiltonian, and, hence, the renormalisation implied by Eq. (89) is generally recommended.

Further, more is possible when the near-identity transformation (89) is enhanced to

$$
\begin{aligned}
B= & A+\mu\left\{\frac{1}{2} a A^{2}+b A_{X X}+a^{\prime} A_{X} \int^{X} A d X\right. \\
& \left.+b^{\prime} X A_{T}\right\} .
\end{aligned}
$$

It can then be shown that assuming $\alpha, \beta$ are not zero, it is possible to choose the available constants $a, b, a^{\prime}, b^{\prime}$ so that the resulting equation for $B$ is just the Korteweg-de Vries equation (i.e. has the form (62), or alternatively, the coefficients $\beta_{1}, \alpha_{1}, \gamma_{1}, \gamma_{2}$ in Eq. (81) are all zero) with an error of $O\left(\mu^{3}\right)$ (see, e.g. Kodama, 1985; Fokas and Liu, 1996; Fokas et al., 1996). Thus, in general, the Eq. (62) is asymptotically reducible to the integrable Korteweg-de Vries equation. However, we hasten to point out that although this is an intriguing result, its use in practice in this context may well be very limited because the amplitude parameter $\alpha$ is not necessarily so small that the transformation (92) is applicable, and also there are circumstances when the coefficient $\alpha$ is zero, in which case this reduction is not possible.

Acknowledgement. EP and OP were supported from INTAS (991068, 99-1637) and RFBR (00-05-64223, 01-05-06208).

\section{Appendix A Coefficients of the extended Korteweg-de Vries equation for internal waves in a two-layer shear flow}

$$
\begin{aligned}
& \alpha=\frac{3}{2} \frac{1}{m h} \frac{\rho_{1} c^{2} m^{3}+\rho_{2}\left(c-U_{0}\right)^{2} h^{2}(H-h)}{\rho_{1} c m^{2}+\rho_{2}\left(c-U_{0}\right) h(H-h)}, \\
& \beta=\frac{h}{6} \quad \cdot \frac{\rho_{1} c^{2} h m^{2}+\rho_{2}\left(c-U_{0}\right)^{2}\left((H-h+m)^{3}-m^{3}\right)}{\rho_{1} c m^{2}+\rho_{2}\left(c-U_{0}\right) h(H-h)}, \\
& \alpha_{1} I=\rho_{1}\left\{\frac{9 a c^{2}}{h}-\frac{6 c^{2}}{h^{3}}-\frac{\alpha^{2}}{h}+\frac{5 \alpha c}{h^{2}}-4 \alpha a c\right\}+\rho_{2}(H-h)\left\{\frac{3\left(c-U_{0}\right)^{2}}{m^{2}}\left[\frac{3 a h}{m}-3 a_{1}-\frac{2}{m^{2}}\right]\right. \\
& \left.-\frac{\alpha^{2}}{m^{2}}+\frac{\alpha\left(c-U_{0}\right)}{m}\left[\frac{5}{m^{2}}-\frac{4 a h}{m}+4 a_{1}\right]\right\}, \\
& \beta_{1} I=\rho_{1}\left\{-\frac{c^{2} h^{3}}{30}+\frac{c^{2} h^{3}}{18}+\frac{2 \beta c h}{3}-\frac{\beta^{2}}{h}\right\}+\rho_{2}\left\{-\frac{\left(c-U_{0}\right)^{2}}{30 m^{2}}\left[(m+H-h)^{5}-m^{5}\right]\right. \\
& +\frac{\left(c-U_{0}\right)}{3 m}\left[a_{2}\left(c-U_{0}\right)+\frac{3 \beta}{m}\right]\left[(m+H-h)^{3}-m^{3}\right]-\frac{\left(c-U_{0}\right)^{2}}{2}\left[-a_{2}+\frac{m}{6}\right]\left[(m+H-h)^{2}-m^{2}\right] \\
& \left.-(H-h)\left[\frac{\beta^{2}}{m^{2}}+2 \beta\left(c-U_{0}\right) \frac{a_{2}}{m}\right]\right\}, \\
& \gamma_{1} I=-\rho_{1}\left\{-\frac{2}{3} \alpha c h+\frac{1}{3} c^{2}-\frac{2}{3} a c^{2} h^{2}+\frac{2 \alpha \beta}{h}+4 \beta a c-\frac{4 \beta c}{h^{2}}\right\} \\
& -\rho_{2}\left\{\frac{\left(c-U_{0}\right)}{3 m}\left[\frac{5\left(c-U_{0}\right)}{2 m^{2}}-\frac{3 \alpha}{m}-2\left(c-U_{0}\right)\left(\frac{a h}{m}-a_{1}\right)\right]\left[(m+H-h)^{3}-m^{3}\right]\right. \\
& -\left(c-U_{0}\right)^{2} a_{1}\left[(m+H-h)^{2}-m^{2}\right] \\
& \left.+\frac{(H-h)}{m}\left[\frac{2 \alpha \beta}{m}-3\left(c-U_{0}\right)^{2} \frac{a_{2}}{m}+2 \alpha\left(c-U_{0}\right) a_{2}+4 \beta\left(c-U_{0}\right)\left(\frac{a h}{m}-a_{1}-\frac{1}{m^{2}}\right)\right]\right\}, \\
& \gamma_{2} I=\rho_{1}\left\{\frac{2 \beta c}{h^{2}}+2 \alpha c h+2 a c^{2} h^{2}-\frac{3 \alpha \beta}{h}-\frac{2 c^{2}}{3}\right\}+\rho_{2}\left\{\frac{\left(c-U_{0}\right)}{m}\left[\frac{3 \alpha}{m}-\frac{7\left(c-U_{0}\right)}{6 m^{2}}+2\left(c-U_{0}\right)\left(\frac{a h}{m}-a_{1}\right)\right]\right. \\
& \cdot\left[(m+H-h)^{3}-m^{3}\right]+3 a_{1}\left(c-U_{0}\right)^{2}\left[(m+H-h)^{2}-m^{2}\right]+\frac{(H-h)}{m}\left[\frac{2 \beta\left(c-U_{0}\right)}{m^{2}}-\frac{3 \alpha \beta}{m}\right. \\
& \left.\left.-6 \alpha\left(c-U_{0}\right) a_{2}+\frac{3\left(c-U_{0}\right)^{2} a_{2}}{m}\right]\right\},
\end{aligned}
$$


where

$$
\begin{aligned}
I & =2 \frac{\rho_{1} c m^{2}+\rho_{2}\left(c-U_{0}\right) h(H-h)}{h m^{2}}, \\
a & =a_{1} \frac{H-h}{h} .
\end{aligned}
$$

\section{Appendix B Numerical calculations for a three-layer profile}

As discussed at the end of Sect. 5 we replaced the two-layer model considered there with a three-layer model, in which the lower layer has a constant density $\rho_{1}$, and the upper layer has a constant density $\rho_{2}$ and a constant current $U_{0}$. The middle layer is thin, with a thickness $\delta$, and has a constant bouyancy frequency $N_{0}$ and a constant current shear $U_{0} / \delta$, both constructed to ensure that the basic density and current profiles are everywhere continuous. Thus, in the middle layer, we put

$\rho(y)=\rho_{0} \exp \left(-\sigma N_{0}^{2} y\right)$

so that

$N(y)=N_{0}$

where

$N_{0}^{2}=\frac{1}{\sigma \delta} \ln \frac{\rho_{1}}{\rho_{2}}$

and

$$
\begin{aligned}
& \rho_{0}=\rho_{2} \exp \frac{1}{\delta} \ln \frac{\rho_{1}}{\rho_{2}}\left(h+\frac{\delta}{2}\right) \\
& U(y)=\frac{U_{0}}{\delta}\left(y-h+\frac{\delta}{2}\right) .
\end{aligned}
$$

The parameters of this three-layer model are $\rho_{1} / \rho_{2}=1.5$, $\delta=H / 100=0.5 \mathrm{~m}, U_{0}=c / 4$, and $N_{0}=1.42$ $1 / \mathrm{s}$. The Richardson number in the middle layer is given by $R i=\left\{\delta \ln \left(\rho_{1} / \rho_{2}\right)\right\} / \sigma U 0^{2}$, which varies with $h / H$, since $c$ then varies and so correspondingly does $U_{0}$. We took $\max (c)=3.84 \mathrm{~m} / \mathrm{s}$, and then $R i=0.55$. For this case the coefficients $\alpha, \beta, \alpha_{1}, \beta_{1}, \gamma_{1}$ and $\gamma_{2}$ of the extended Korteweg-de Vries equation were evaluated numerically, and the results are shown in Fig. 4.

\section{Appendix C Coefficients of the extended Korteweg-de Vries equation for surface waves on a two-layer shear flow}

Here we consider the surface wave mode for the two-layer shear flow, described at the beginning of Sect. 5. The procedure is similar to that for the internal wave mode, one important difference being that there is a different normalisation. The modal function is again found from Eqs. (50), (51), and (52), and is now given by
$\Phi(y)=b \begin{cases}y / h, & 0 \leq y<h \\ (y+m-h) / m, & h \leq y \leq H\end{cases}$

where

$b=\frac{m}{m+H-h}$

and

$m=\sigma\left(c-U_{0}\right)^{2}+h-H$.

The linear long-wave phase speed $c$ is a solution of Eq. (85). The phase speed for surface mode can be expressed in the semi-explicit form

$c^{2}=\frac{(2+\sigma)\left(H-h+(1-u)^{2} h\right)+\sqrt{D}}{2(1-u)^{2} \sigma(2+\sigma)}$,

where

$$
\begin{aligned}
D= & (2+\sigma)\left[(2+\sigma)\left(H-h+(1-u)^{2} h\right)^{2}\right. \\
& \left.-8(1-u)^{2} \sigma h(H-h)\right],
\end{aligned}
$$

and $u=U_{0} / c$ is the relative shear flow velocity.

In the limiting cases, when the thickness of one layer tends to zero thus reducing the model to a one-layer unstratified fluid, the phase speed $c$ becomes

$c(h \rightarrow H) \rightarrow \sqrt{\frac{H}{\sigma}}$

$c(h \rightarrow 0) \rightarrow \frac{1}{1-u} \sqrt{\frac{H}{\sigma}}=\sqrt{\frac{H}{\sigma}}+U_{0}$.

In dimensional variables (using Eqs. 8 and 9) these formulas transform to the usual expressions for the linear longwave phase speed of a surface wave on an unstratified fluid of depth $H$, i.e. $\sqrt{g H}$ and $\sqrt{g H}+U_{0}$, respectively. Note that $c$, or $c-U_{0}$ scale with $\sigma^{-1 / 2}$, and keeping this in mind we see that it is then permissible to take the limit $\sigma \rightarrow 0$ to obtain the surface wave mode in an unstratified fluid.

Next we use Eqs. (68), (69), (70), (71), and (72) to find the nonlinear and dispersion corrections to the modal function for this surface wave mode. The nonlinear correction has the form

$T_{n}(y)=\left\{\begin{array}{cc}a y, & 0 \leq y<h \\ \left(\frac{a h}{m}-a_{2}\right)(y+m-h) & \\ +a_{2} m, & h \leq y \leq H\end{array}\right.$

where

$a=\frac{H-h}{h} \frac{1}{\sigma\left(c-U_{0}\right)^{3}}\left(\alpha-\frac{3}{2} \frac{1}{\sigma\left(c-U_{0}\right)}\right)$

and

$a_{2}=p \sigma\left(c-U_{0}\right) \frac{b}{m^{2}}\left(\alpha-\frac{3}{2}\left(c-U_{0}\right) \frac{b}{m}\right)$.

The dispersion correction has the form 
$T_{d}(y)= \begin{cases}-\frac{b y^{3}}{6 h}+a_{1} y, & 0 \leq y<h \\ -\frac{b(y-h+m)^{3}}{6 m}+a_{11}(y-h)+a_{1} h+b \frac{m^{2}-h^{2}}{6}, & h \leq y \leq H\end{cases}$

where

$a_{11}=-p \sigma\left(c-U_{0}\right) \frac{b}{m^{2}}\left(2 \beta-\frac{1}{2}\left(c-U_{0}\right)(m+H-h)^{2}\right)++\frac{b}{6 m}\left(m^{2}-h^{2}-\frac{(m+H-h)^{3}}{m}\right)+a_{1} \frac{h}{m}$

and

$a_{1}=\frac{b}{h}\left(\frac{1}{6}\left(\frac{(m+H-h)^{3}}{m}-m^{2}+h^{2}\right)+\frac{b \sigma\left(c-U_{0}\right)(H-h)}{m^{2}}\left(2 \beta-\frac{1}{2}\left(c-U_{0}\right)(m+H-h)^{2}\right)\right)$.

Next, from Eqs. (63), (64), (76), (77), (78), and (79) we can evaluate the coefficients of the extended Korteweg-de Vries equation (81) and find that here,

$$
\begin{aligned}
& \alpha= \frac{3}{2} \frac{b}{m h} \frac{\rho_{1} c^{2} m^{3}+\rho_{2}\left(c-U_{0}\right)^{2} h^{2}(H-h)}{\rho_{1} c m^{2}+\rho_{2}\left(c-U_{0}\right) h(H-h)} \\
& \beta= \frac{h}{6} \frac{\rho_{1} c^{2} h m^{2}+\rho_{2}\left(c-U_{0}\right)^{2}\left((H-h+m)^{3}-m^{3}\right)}{\rho_{1} c m^{2}+\rho_{2}\left(c-U_{0}\right) h(H-h)}, \\
& \alpha_{1} I= \rho_{1}\left\{\frac{9 a b^{2} c^{2}}{h}-\frac{6 b^{4} c^{2}}{h^{3}}-\frac{\alpha^{2} b^{2}}{h}+\frac{5 \alpha b^{3} c}{h^{2}}-4 \alpha a b c\right\} \\
&+\rho_{2}(H-h)\left\{3\left(c-U_{0}\right)^{2} \frac{b^{2}}{m^{2}}\left[\frac{3 a h}{m}-3 a_{2}-\frac{2 b^{2}}{m^{2}}\right]-\frac{\alpha^{2} b^{2}}{m^{2}}+\alpha\left(c-U_{0}\right) \frac{b}{m}\left[\frac{5 b^{2}}{m^{2}}-\frac{4 a h}{m}+4 a_{2}\right]\right\} \\
& \beta_{1} I= \rho_{1}\left\{-\frac{b^{2} c^{2} h^{3}}{30}+\frac{a_{1} b c^{2} h^{2}}{3}+\beta c b^{2} h-\frac{\beta^{2} b^{2}}{h}-2 \beta a_{1} c b\right\}+\rho_{2}\left\{-\frac{\left(c-U_{0}\right)^{2} b^{2}}{30 m^{2}}\left[(m+H-h)^{5}-m^{5}\right]\right. \\
& \quad+\left(c-U_{0}\right) \frac{b}{3 m}\left[a_{11}\left(c-U_{0}\right)+\frac{3 \beta b}{m}\right]\left[(m+H-h)^{3}-m^{3}\right] \\
&-\left(c-U_{0}\right)^{2} \frac{b}{2 m}\left[-a_{11} m+a_{1} h+b \frac{m^{2}-h^{2}}{6}\right]\left[(m+H-h)^{2}-m^{2}\right] \\
&\left.\quad-(H-h)\left[\frac{\beta^{2} b^{2}}{m^{2}}+2 \beta\left(c-U_{0}\right) \frac{a_{11} b}{m}\right]\right\} \\
& \gamma_{1} I=-\rho_{1}\left\{-\alpha b^{2} c h+\frac{5}{6} c^{2} b^{3}-\frac{2}{3} a b c^{2} h^{2}+\frac{2 \alpha \beta b^{2}}{h}-\frac{3 a_{1} b^{2} c^{2}}{h}+2 \alpha a_{1} b c+4 \beta a b c-\frac{4 \beta b^{3} c}{h^{2}}\right\}
\end{aligned}
$$$$
-\rho_{2}\left\{\left(c-U_{0}\right) \frac{b}{3 m}\left[\frac{5\left(c-U_{0}\right) b^{2}}{2 m^{2}}-\frac{3 \alpha b}{m}-2\left(c-U_{0}\right)\left(\frac{a h}{m}-a_{2}\right)\right]\left[(m+H-h)^{3}-m^{3}\right]\right.
$$$$
-\left(c-U_{0}\right)^{2} a_{2} b\left[(m+H-h)^{2}-m^{2}\right]
$$$$
\left.+(H-h) \frac{b}{m}\left[\frac{2 \alpha \beta b}{m}-3\left(c-U_{0}\right)^{2} \frac{a_{11} b}{m}+2 \alpha\left(c-U_{0}\right) a_{11}+4 \beta\left(c-U_{0}\right)\left(\frac{a h}{m}-a_{2}\right)-4 \beta\left(c-U_{0}\right) \frac{b^{2}}{m^{2}}\right]\right\}
$$$$
\gamma_{2} I=\rho_{1}\left\{\frac{2 \beta b^{3} c}{h^{2}}+3 \alpha b^{2} c h+2 a b c^{2} h^{2}-\frac{3 \alpha \beta b^{2}}{h}-\frac{7 b^{3} c^{2}}{6}+\frac{3 a_{1} b^{2} c^{2}}{h}-6 \alpha a_{1} b c\right\}
$$$$
+\rho_{2}\left\{\left(c-U_{0}\right) \frac{b}{m}\left[\frac{3 \alpha b}{m}-\frac{7\left(c-U_{0}\right) b^{2}}{6 m^{2}}+2\left(c-U_{0}\right)\left(\frac{a h}{m}-a_{2}\right)\right]\left[(m+H-h)^{3}-m^{3}\right]\right.
$$$$
+3 a_{2} b\left(c-U_{0}\right)^{2}\left[(m+H-h)^{2}-m^{2}\right]
$$$$
\left.+\frac{b}{m}(H-h)\left[2 \beta\left(c-U_{0}\right) \frac{b^{2}}{m^{2}}-\frac{3 \alpha \beta b}{m}-6 \alpha\left(c-U_{0}\right) a_{11}+3\left(c-U_{0}\right)^{2} a_{11} \frac{b}{m}\right]\right\},
$$ 
where

$$
I=2 b^{2} \frac{\rho_{1} c m^{2}+\rho_{2}\left(c-U_{0}\right) h(H-h)}{h m^{2}} .
$$

Without a shear flow and density stratification we have only the familiar surface wave mode, which can be obtained from the above formulae by one of several limits, e.g. letting $h \rightarrow H$. In this case the coefficients $\alpha, \beta, \alpha_{1}, \beta_{1}, \gamma_{1}$, $\gamma_{2}$ reduce to the well-known expressions for surface waves in unstratified water of depth $H$ (see, for instance, Marchant and Smyth (1990),

$$
\begin{aligned}
\alpha=\frac{3}{2} \frac{c}{H}, & \beta=\frac{1}{6} c H^{2}, & \alpha_{1}=-\frac{3}{8} \frac{c}{H^{2}}, \\
\beta_{1}=\frac{19}{360} c H^{4}, & \gamma_{1}=\frac{5}{12} c H, & \gamma_{2}=\frac{23}{24} c H .
\end{aligned}
$$

\section{References}

Benney, D. J.: Long nonlinear waves in fluid flows, J. Math. Phys., 45, 52-63, 1966.

Benney, D. J., and Ko, D. R. S.: The propagation of long large amplitude internal waves, Stud. Appl. Math., 59, 187-199, 1978.

Fokas, A. and Liu, Q. M.: Asymptotic integrability of water waves, Phys. Rev. Lett., 77, 2347-2351, 1996.

Fokas, A., Grimshaw, R. H. J., and Pelinovsky, D. E.: On the asymptotic integrability of a higher-order evolution equation describing internal waves in a deep fluid, J. Math. Phys., 37, 34153421, 1996.

Funakoshi, M.: Long internal waves in a two-layer fluid, J. Phys. Soc. Japan, 54, 2470-2476, 1985.

Funakoshi, M. and Oikawa, M.: Long internal waves of large amplitude in a two-layer fluid, J. Phys. Soc. Japan, 55, 128-144, 1986.

Gear, J. A. and Grimshaw, R.: A second- order theory for solitary waves in shallow fluids, Phys. Fluids, 26, 14-29, 1983.

Grimshaw, R.: Internal solitary waves, in: Advances in Coastal and Ocean Engineering, (Ed) Liu, P. L.-F., World Scientific Publishing Company, Singapore, 3, 1-30, 1997.
Grimshaw, R., Pelinovsky, E. and Talipova, T.: The modified Korteweg-de Vries equation in the theory of the large amplitude internal waves, Non. Proc. Geophys., 4, 237-250, 1997.

Holloway, P., Pelinovsky, E., Talipova, T., and Barnes, B.: A nonlinear model of internal tide transformation on the Australian NorthWest Shelf, J. Phys. Oceanography, 27, 871-896, 1997.

Holloway, P., Pelinovsky, E. and, Talipova, T.: A generalised Korteweg-de Vries model of internal tide transformation in the coastal zone, J. Geophys. Res., 104, 18 333-18 350, 1999.

Kakutani, T. and Yamasaki, N.: Solitary waves in a two-layer fluid, J. Phys. Soc. Japan, 45, 674-679, 1978.

Kodama, Y.: Nearly integrable systems. Physica D, 16, 14-26, 1985.

Koop, C. G. and Butler, G.: An investigation of internal solitary waves in a two-fluid system, J. Fluid Mech., 112, 225-251, 1981.

Lamb, K. and Yan, L.: The evolution of internal wave undular bores: comparisons of a fully nonlinear numerical model with weakly nonlinear theory, J. Phys. Oceanography, 26, 2712-2734, 1996.

Lee, C. and Beardsley, R. C.: The generation of long nonlinear internal waves in a weakly stratified shear flow, J. Geophys. Res., 79, 453-462, 1974.

Long, R. R.: Solitary waves in one- and two-fluids systems, Tellus, 8, 460-471, 1956.

Marchant, T. R. and Smyth, N. F.: The extended Korteweg-de Vries equation and the resonant flow over topography, J. Fluid Mech., 221, 263-288, 1990.

Pelinovsky, E., Talipova, T., and Stepanyants, Yu.: Modelling of the propagation of nonlinear internal internal waves in horizontally inhomogeneous ocean, Izv. Atm. Oceanic Phys., 30, 79-85, 1994.

Pelinovsky, E. N., Poloukhina, O. E., and Lamb, K.: Nonlinear internal waves in the ocean stratified on density and current, Oceanology, 40, 805-815, 2000.

Prasad, D. and Akylas, T. R.: On the generation of shelves by long nonlinear waves in stratified flows, J. Fluid Mech., 346, 345-362, 1997.

Segur, H. and Hammack, J. L.: Soliton models of long internal waves, J. Fluid Mech., 118, 285-304, 1982.

Talipova, T., Pelinovsky, E., Lamb, K., Grimshaw, R., and Holloway, P.: Cubic effects at the intense internal wave propagation, Doklady Earth Sciences, 365, 241-244, 1999. 